\title{
Multi-scale simulation of three-dimensional thin-film lubrication
}

\author{
Zuobing WU $\mathbf{W}^{1,2 *}$ \\ ${ }^{1}$ State Key Laboratory of Nonlinear Mechanics, Institute of Mechanics, Chinese Academy of Sciences, Beijing 100190, China \\ ${ }^{2}$ School of Engineering Science, University of Chinese Academy of Sciences, Beijing 100049, China \\ Received: 02 November 2019 / Revised: 25 November 2019 / Accepted: 04 December 2019 \\ (C) The author(s) 2019.
}

\begin{abstract}
For three-dimensional (3D) mono-layer molecular thin-film lubrication, the elasticity of the substrate affects the tribological behaviors of a thin fluid film confined by two solid substrates. To account for the elastic effects, this study establishes a multi-scale method that combines an atomistic description of the near region with a coarse-grained description of the far region of the solid substrate to simulate the thin-film lubrication. It is demonstrated that for a given temperature range and film-substrate coupling strength, the multi-scale method is in excellent agreement with the fully atomistic simulation. This study reveals that the elastic response of the substrate can be effectively rendered in the hybrid scheme. In the application of the multi-scale method to investigate the tribological properties of the multi-layer molecular thin-film lubrication, it is determined that the systematic static friction coefficient monotonously decreases as the molecular layer thickness in the fluid film increases. In comparison to the mono-layer molecular thin-film lubrication, the multi-layer molecular thin-film lubrication plays a role in reducing the friction and wear of the system.
\end{abstract}

Keywords: thin-film lubrication; film-substrate coupling strength; multi-scale method; friction

\section{Introduction}

Thin-film lubrication describes the relative sliding of two solid substrates that are separated by a thin fluid film. As a prototype tribological system, thin-film lubrication plays a fundamental role in the development of advanced ultra-precision mechanical equipment and micro-machines [1-4]. At the interface, the surfaces of the solid substrates appear to be perfectly smooth on a macroscopic scale. The thin fluid film can protect the surfaces from being damaged when the surfaces are sheared and reduce the friction force or the friction coefficient of the system, in comparison with dry friction. However, on the microscopic scale, the substrates make molecular contact with relatively few asperities and they undergo elastic deformation and plastic flow. The fluid molecules are adsorbed and adhered to the solid surfaces. Based on the crystalline model, in which a mono-layer fluid film is confined between two solid walls that are composed of crystal atoms, the solid-liquid transitions of the film or the stick-slip processes of the system have been identified $[5,6]$. The nonlinear relationship between the static friction force and the load was determined, which depends on the structures of the surfaces and the coverage of the film [7, 8]. For a multi-layer fluid film, rheological changes occur for the confined fluid due to its bulk behavior. This includes enhancing its equivalent viscosity, forming its ordering structure, and the solid-liquid transition $[9,10]$. It is clearly reasonable that thin-film lubrication contains a complicated interplay between the multiple spatial scales that ranges from the macroscale to the molecular scale [11-15].

To gain deeper insights into thin-film lubrication, it is necessary to know the various spatial scale couplings at the interface and those far away from the interface. The coupling at the interface includes large amplitude

* Corresponding author: Zuobing WU, E-mail: wuzb@lnm.imech.ac.cn 
diffusive motions of the fluid molecules and the solid atoms of the substrates near the interface, which strongly influences the static frictional force. The fluid film and the near regions of the solid substrates must be described atomistically to reflect the thermomechanical properties. Since the solid atoms of the substrates that are far removed from the interface slightly oscillate at their equilibrium positions, the far regions of the solid substrates undergo elastic distortion. As a result, the far regions can be coarsegrained and treated as a macroscale (continuum). Therefore, the fine distinctions of the atomic motions are submerged; however, the global description of these interesting processes need to be further explored. The scientific challenge is how to meld an atomistic description that is necessary to treat the highly heterogeneous interfacial region with a continuum description that is adequate for the bulk region of the system.

During the past three decades, a series of coarsegraining methods have been developed to analyze the crack propagation in solids, complex fluid flow, and the evolution of biological macromolecules [16-19]. A prominent quasistatic multi-scale approach is the quasicontinuum method, in which the whole lattice of the materials is coarsely divided by overlaying it with a finite element mesh to treat the quasistatic evolution of the defects at zero temperature [20]. It eliminates the myriad of the original atoms in favor of fewer nodes in the mesh. With the correction of free energy on the non-nodal atoms of the mesh, an extension of the quasicontinuum method to treat solid systems at a nonzero temperature has been developed $[21,22]$. The extended quasicontinuum technique or the free-energy corrected hybrid atomistic and coarse-grained method was then successfully applied in two-dimensional thin-film lubrication [23, 24]. Moreover, a hybrid atomistic/continuum modeling for two-dimensional contact and dry friction between the solid substrates has been proposed [25, 26]. Recently, for three-dimensional (3D) contact, a multi-scale coupling of the molecular dynamics and the finite element method has been reported [27]. However, to the best of our knowledge, multi-scale simulation of 3D thinfilm lubrication considering the effects of elastic substrates has not been reported.
This study developed a multi-scale method to treat 3D thin-film lubrication and to account for the effects of the elastic substrates. Section 2 describes the mono-layer molecular thin-film lubrication system and the multi-scale method. Section 3 provides the computational procedure and the parameters of the system. The numerical results of the thin-film lubrication system are obtained by using the fully atomistic, several approximate, and the multi-scale methods; these are analyzed in Section 4. Meanwhile, the validity of the multi-scale method for a specific temperature range, film-substrate coupling strength, and its application in multi-layer molecular thin-film lubrication systems are further presented. Finally, Section 5 presents the discussion and conclusions for this study.

\section{$23 \mathrm{D}$ thin film lubrication}

\subsection{Fully atomic description}

Figure 1(a) shows a schematic diagram of the simplified 3D contact, which consists of a face-centered cubic (FCC) crystalline substrate and a mono-layer molecular thin-film. It can be thought as an extension of the previous crystalline model. The upper substrate is assumed elastic, while the lower substrate is still rigid and is reduced to the bottom wall due to the consideration of the timesaving computation (it is straightforward to extend the model with two elastic substrates). The top and bottom walls are assumed to be rigid and remain crystallographically aligned within the FCC (001) plane. The bottom wall remains stationary in the "laboratory" reference frame; the top wall can be shifted in the $x$ - and $y$-directions. The top wall remains parallel with the bottom wall and it serves as a handle in which the substrate can be manipulated. Its sliding distance in the $x$-direction is specified by the registry $\alpha$, which is defined as

$$
x_{i}^{\mathrm{t}}=x_{i}^{\mathrm{b}}+\alpha a
$$

where $x_{i}^{\mathrm{t}}$ and $x_{i}^{\mathrm{b}}$ denote the lateral positions of the atoms in the top and bottom walls, respectively. $\alpha$ is the distance in the unit of the lattice constant in which the top wall is displaced laterally with respect to the bottom wall. The total configurational energy 


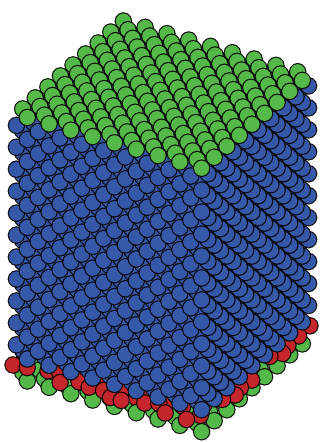

(a)

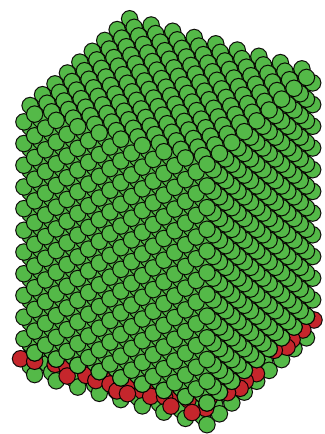

(c)

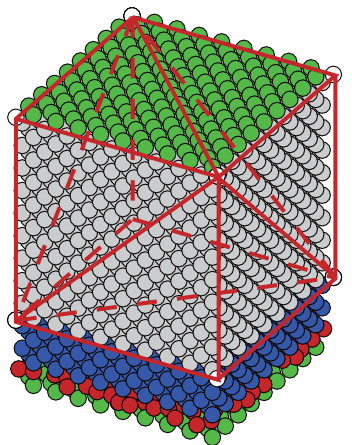

(b)

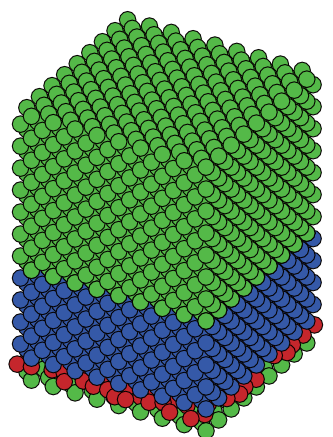

(d)
Fig. 1 Schematic diagram of 3D mono-layer molecular thin-film lubrication system with periodic boundary conditions in the $x$ and $y$ directions. The wall, the elastic/rigid substrate, and the fluid atoms are represented by the green, blue/green, and red spheres, respectively. (a) Fully atomistic description, (b) partial coarse graining of the far region of the substrate, (c) wholly rigid substrate/0-layer near the region or the crystalline model, and (d) rigid far region/8-layer near region. The coarse-grained far region of the substrate is represented by the gray spheres. All atoms are depicted in their initial configuration at $\alpha=0$.

of the system is then given by

$$
\begin{aligned}
U\left(\mathbf{r}^{N_{\mathrm{f}}}, \mathbf{r}^{N_{\mathrm{s}}}\right)= & \frac{1}{2} \sum_{i=1}^{N_{f}} \sum_{j \neq i}^{N_{f}} u_{\mathrm{ff}}\left(r_{i j}\right)+\sum_{i=1}^{N_{f}} \sum_{j=1}^{N_{\mathrm{s}}} u_{\mathrm{fs}}\left(r_{i j}\right) \\
& +\frac{1}{2} \sum_{i=1}^{N_{\mathrm{s}}} \sum_{j=1}^{N_{\mathrm{s}}} u_{\mathrm{ss}}\left(r_{i j}\right)+\sum_{i=1}^{N_{f}} \sum_{j=1}^{N_{\mathrm{w}}} u_{\mathrm{fs}}\left(r_{i j}\right) \\
& +\sum_{i=1}^{N_{\mathrm{s}}} \sum_{j=1}^{N_{\mathrm{w}}} u_{\mathrm{ss}}\left(r_{i j}\right)+\sum_{i=1}^{N_{\mathrm{w}}^{t}} \sum_{j=1}^{N_{\mathrm{w}}^{\mathrm{b}}} u_{\mathrm{ss}}\left(r_{i j}\right)
\end{aligned}
$$

where $\mathbf{r}^{N_{f}}$ and $\mathbf{r}^{N_{s}}$ represent the collections of the $3 N_{\mathrm{f}}$ fluid-atom coordinates in the film and the $3 N_{\mathrm{s}}$ solid-atom coordinates in the substrate. Note that $U$ depends on the $3 N_{\mathrm{w}}=3\left(N_{\mathrm{w}}^{\mathrm{t}}+N_{\mathrm{w}}^{\mathrm{b}}\right)$ coordinates of the wall atoms through the last three terms in Eq. (2). The pair interactions are taken to be shifted by the Lennard-Jones potentials as follows:

$$
\begin{gathered}
u_{\mathrm{ab}}(r)=\left\{\begin{array}{lll}
\phi_{\mathrm{ab}}(r)-\phi_{\mathrm{ab}}\left(r_{\mathrm{c}}\right), & \text { if } & r<r_{\mathrm{c}} \\
0, & \text { if } & r \geq r_{\mathrm{c}}
\end{array}\right. \\
\phi_{\mathrm{ab}}(r)=4 \varepsilon_{\mathrm{ab}}\left[(\sigma / r)^{12}-(\sigma / r)^{6}\right], \mathrm{ab}=\mathrm{ff}, \mathrm{fs}, \mathrm{ss}
\end{gathered}
$$

where the effective diameter $\sigma$ and range $r_{\mathrm{c}}$ are the same for all pairs. Only the depth $\varepsilon_{\mathrm{ab}}$ of the attractive well depends on the composition of the pair.

\subsection{Multi-scale description}

Figure 1(b) displays a schematic of the hybrid atomistic and coarse-grained treatment of the mono-layer molecular thin-film lubrication. The substrate connected with the top wall is divided into the near and far regions, which are depicted by using the atomistic and coarse-grained descriptions, respectively. The coarsegrained far region is covered with a finite element mesh and it is connected to the near region of the substrate. The finite element mesh in Fig. 1(b) consists of the "local" tetrahedral elements, whose nodes coincide with a subset of solid substrates and wall atoms. For the local element, where a cut-off sphere is placed at its "centroid" atom (i.e., the atom closest to the center of the inscribed sphere of the element) is embedded itself, the "ghost force" will not appear in the coarse-graining process [20]. In a tetrahedral element, the center of the inscribed sphere is written as

$$
r_{\mathrm{c} \alpha}=\sum_{k=1}^{4} R_{k \alpha} A_{k} / \sum_{k=1}^{4} A_{k}
$$

where $k$ is the index of the nodes, $R_{k \alpha}$ is the nodal coordinates of the element, and $A_{k}$ is the surface area of the element opposite the node $k$. As shown in Fig. 1(b), the coarse-grained far region of the substrate is divided into five tetrahedral elements, which includes four congruent elements and one larger element (Fig. 2). When an element e is distorted by displacing its nodes, the lattice underlying the element $\mathrm{e}$ is assumed to have a homogeneous deformation. In the tetrahedral element, the coordinates $r_{i \alpha}$ of the underlying atom $i$ can be written in terms of $R_{k \alpha}$ as

$$
r_{i \alpha}=\sum_{k=1}^{4} V_{i}(k) R_{k \alpha} / V_{\mathrm{e}}, \alpha=x, y, z
$$

where $V_{\mathrm{e}}$ and $V_{i}(k)$ denote the volumes of the element e and the inscribed tetrahedron, respectively, which 


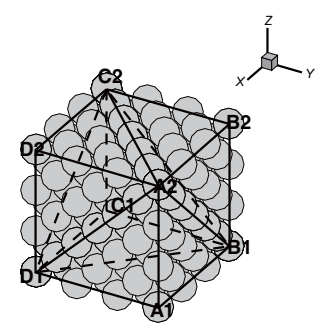

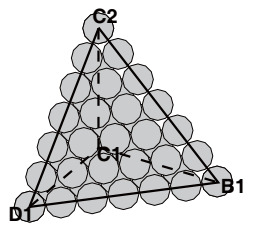

(1)

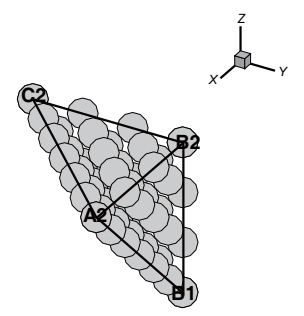

(4)

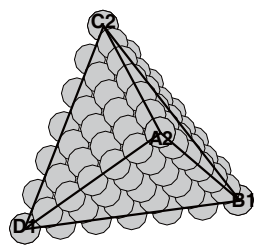

(2)

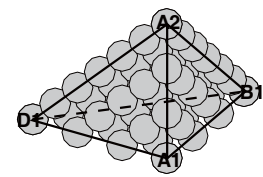

(5)

Fig. 2 Schematic diagram of dividing a cube to constitute a coarse-graining mesh in the far region of the substrate. The tetrahedral elements (1), (3), (4), and (5) are congruent and are smaller than the tetrahedral element (2).

has one surface coinciding with the surface of the element e opposite to the node $k$ and the vertex coinciding with the lattice site at $\left(r_{i x}, r_{i y}, r_{i z}\right)$.

The coarse-graining description of the original substrate atoms in the far region are partitioned into two subsets: $N_{\mathrm{n}}$ nodal atoms and $N_{q}$ non-nodal atoms. Under the assumption of the homogeneous deformation of the element, the contribution of the non-nodal atoms to the free-energy can be transformed into the effective configuration energy of the coarse-grained system. By integrating the Boltzmann factor over the $3 N_{q}$ degrees of freedom of the non-nodal atoms, the effective configurational energy governing the motion of the nodal atoms can be written as [22]

$$
V_{\text {eff }}\left(\mathbf{R}^{N_{\mathrm{n}}}\right)=\sum_{e=1}^{N_{e}}\left(N_{\mathrm{a}}^{\mathrm{e}} \tilde{u}_{\mathrm{e}}+N_{q}^{\mathrm{e}} f_{e}\right)
$$

where $\mathbf{R}^{N_{n}}$ stands for the nodal configuration, $N_{\mathrm{e}}$ represents the number of elements, and $N_{\mathrm{a}}^{\mathrm{e}}\left(N_{q}^{\mathrm{e}}\right)$ is the number of atoms (non-nodal atoms) that are underlying element e. The configurational energy per atom $\tilde{u}_{\mathrm{e}}$ is expressed as

$$
\tilde{u}_{\mathrm{e}}=\frac{1}{2} \sum_{j \neq i} u_{\mathrm{ss}}\left(r_{i j}\right)
$$

where $i$ denotes the centroid atom and $j$ represents the atoms lying within the sphere of radius $r_{\mathrm{c}}$ that is centered on $i$. The Helmholtz energy per non-nodal atom $f_{\mathrm{e}}$ is estimated by using the local harmonic approximation [28] and it is written as

$$
f_{\mathrm{e}}=3 k_{\mathrm{B}} \ln \left[\hbar|D|^{1 / 6} / k_{\mathrm{B}} T\right]
$$

where $k_{\mathrm{B}}$ is Boltzmann's constant, $\hbar$ is Planck's constant, and $T$ is the absolute temperature. The elements of the $3 \times 3$ dynamical matrix are given by

$$
(D)_{k l}=m^{-1}\left(\partial^{2} \tilde{u}_{\mathrm{e}} / \partial x_{k} \partial x_{l}\right)_{0}(k, l=1,2,3)
$$

In addition, $k$ and $l$ represent the Cartesian components $\left(x_{1}=x, x_{2}=y, x_{3}=z\right)$ of the position of the reference atom, and the subscript 0 signifies that the partial derivative is evaluated from the equilibrium configuration.

The total configurational energy of the hybrid system is then given by

$$
\begin{aligned}
U_{\mathrm{c}}\left(\mathbf{r}^{N_{\mathrm{f}}}, \mathbf{r}^{N^{\prime} \mathrm{s}}, \mathbf{R}^{N_{\mathrm{n}}}\right)= & \frac{1}{2} \sum_{i=1}^{N_{f}} \sum_{j \neq i}^{N_{f}} u_{\mathrm{ff}}\left(r_{i j}\right)+\sum_{i=1}^{N_{f}} \sum_{j=1}^{N_{\mathrm{w}}^{\mathrm{b}}} u_{\mathrm{fs}}\left(r_{i j}\right) \\
& +\sum_{i=1}^{N_{f}} \sum_{j=1}^{N^{\prime} s} u_{\mathrm{fs}}\left(r_{i j}\right)+\frac{1}{2} \sum_{i=1}^{N^{\prime} s} \sum_{j \neq i}^{N^{\prime} s} u_{\mathrm{ss}}\left(r_{i j}\right) \\
& +\sum_{i=1}^{N^{\prime} s} \sum_{j=1}^{N_{\mathrm{w}}^{\mathrm{b}}} u_{\mathrm{ss}}\left(r_{i j}\right)+\frac{1}{2} \sum_{i=1}^{N^{\prime} s} \sum_{j=1}^{N^{\prime \prime s}} u_{\mathrm{ss}}\left(r_{i j}\right) \\
& +\frac{1}{2} \sum_{i=1}^{N_{\mathrm{w}}^{\mathrm{t}}} \sum_{j=1}^{N^{\prime \prime s}} u_{\mathrm{ss}}\left(r_{i j}\right)+\sum_{\mathrm{e}=1}^{N_{e}} N_{\mathrm{a}}^{\mathrm{e}}\left[\frac{1}{2} \sum_{j \neq i} u_{\mathrm{ss}}\left(r_{i j}\right)\right] \\
& +\sum_{\mathrm{e}=1}^{N_{e}} N_{q}^{\mathrm{e}} 3 k_{\mathrm{B}} T \ln \left[\hbar|D|^{1 / 6} / k_{\mathrm{B}} T\right]
\end{aligned}
$$


where $N^{\prime}{ }_{s}$ and $N^{\prime \prime}{ }_{s}$ represent the numbers of atoms in the near and far regions of the substrate, respectively. The fluid-fluid and fluid-solid interactions are described in the first three terms in Eq. (11). The band of the near region is sufficiently wide; hence, the fluid atoms do not interact with the underlying atoms in the far region. The solid-solid interaction in the near region atoms and the solid-solid interactions of the near region atoms with the bottom wall atoms and the far region atoms are denoted in the next three terms in Eq. (11). The solid-solid interaction between the far region atoms and the top wall atoms is included in the seventh term in Eq. (11). It should be noted that only half of the interaction in the sixth/seventh term in Eq. (11) is accounted for. The coarse-grained contribution in the next to last term in Eq. (11) effectively accounts for the other half of the interactions that are between the underlying and near-region atoms/top wall atoms.

\subsection{Statistical thermodynamic analysis}

To compute the thermomechanical properties, this study employed the analogue of the isothermalisobaric ensemble and took the usual Gibbs free energy $G$ as its characteristic function, which is described by

$$
G=E-T S-A L_{z} T_{z z}
$$

where $E$ represents the internal energy, $S$ is the entropy, $A$ is the area of the system in the $x-y$ plane, $L_{z}$ is the vertical distance between the two walls, and $T_{z z}$ is the normal stress. The change in $G$ under a reversible transformation of the system is written as $[29,30]$

$$
\mathrm{d} G=-S \mathrm{~d} T+\mu \mathrm{d} N_{\mathrm{f}}+\gamma \mathrm{d} A-A L_{z} \mathrm{~d} T_{z z}+T_{z x} A \mathrm{~d}(\alpha a)
$$

where $\mu$ is the chemical potential of the fluid, $\gamma$ is the interfacial tension, and $T_{z x}$ is the shear stress.

By performing a standard statistical-mechanical analysis [31, 32], we can obtain the following relationship of the characteristic function $G$ with the isothermal-isobaric partition function $\Delta$ :

$$
\begin{gathered}
G=-k_{\mathrm{B}} T \ln \Delta \\
\Delta\left(T, N_{\mathrm{f}}, T_{z z}, \alpha a\right)=\sum_{L_{z}} \exp \left[\left(A L_{z}\right) T_{z z} / k_{\mathrm{B}} T\right] Q_{N_{\mathrm{f}}}
\end{gathered}
$$

In Eq. (15), the canonical partition function $Q_{N_{f}}$ can be expressed as

$$
Q_{N_{\mathrm{f}}}=\frac{1}{h^{3\left(N_{\mathrm{f}}+N_{\mathrm{s}}\right)}} \frac{1}{N_{\mathrm{f}} !} \int \mathrm{d} \mathbf{p}^{N_{\mathrm{f}}} \int \mathrm{d} \mathbf{r}^{N_{\mathrm{f}}} \int \mathrm{d} \mathbf{p}^{N_{\mathrm{s}}} \int \mathrm{d} \mathbf{r}^{N_{\mathrm{s}}} \exp \left(-H / k_{\mathrm{B}} T\right)
$$

where $h$ is Planck's constant. The Hamiltonian $H$ is written as

$$
H=\sum_{i=1}^{N_{f}} \mathbf{p}_{i}^{2} / 2 m_{\mathrm{f}}+\sum_{i=1}^{N_{\mathrm{s}}} \mathbf{p}_{i}^{2} / 2 m_{\mathrm{s}}+U\left(\mathbf{r}^{N_{\mathrm{f}}}, \mathbf{r}^{N_{\mathrm{s}}}\right)
$$

where $\mathbf{p}^{N_{f}}$ and $\mathbf{p}^{N_{s}}$ represent the momenta conjugate to the coordinates $\mathbf{r}^{N_{f}}$ and $\mathbf{r}^{N_{s}}$, respectively. After the integral for the momenta, Eq. (16) can be rewritten as

$$
Q_{N_{f}}=\frac{1}{N_{f} !} \Lambda_{f}^{3 N_{f}} \Lambda_{s}^{3 N_{s}} Z_{N_{f}}
$$

where $\Lambda_{\mathrm{f}}=\sqrt{2 \pi m_{\mathrm{f}} T} / h, \Lambda_{\mathrm{s}}=\sqrt{2 \pi m_{\mathrm{s}} T} / h$, and the configuration integral $Z_{N_{\mathrm{f}}}$ is

$$
Z_{N_{\mathrm{f}}}=\int \mathrm{d} \mathbf{r}^{N_{\mathrm{f}}} \int \mathrm{d} \mathbf{r}^{N_{\mathrm{s}}} \exp \left(-U / k_{\mathrm{B}} T\right)
$$

From Eqs. (13)-(17), the shear stress can be obtained as follows:

$$
\begin{aligned}
A T_{z x} & =\frac{\partial G}{\partial \alpha a}=-\frac{k_{\mathrm{B}} T}{\Delta} \frac{\partial \Delta}{\partial \alpha a} \\
& =-k_{\mathrm{B}} T \frac{\sum_{L_{z}} \exp \left(A L_{z} T_{z z} / k_{B} T\right) \frac{\partial Z_{N_{f}}}{\partial \alpha a}}{\sum_{L_{z}} \exp \left(A L_{z} T_{z z} / k_{\mathrm{B}} T\right) Z_{N_{\mathrm{f}}}}
\end{aligned}
$$

Using the variable transformation as follows:

$$
x_{i}^{\prime}=x_{i}-\alpha a z_{i} / L_{z}
$$

we can rewrite the configuration integral $Z_{N_{\mathrm{f}}}$ as

$$
Z_{N_{\mathrm{f}}}=\prod_{i=1}^{N_{\mathrm{f}}+N_{\mathrm{s}}} \int \mathrm{d} \mathbf{r}^{N_{f}} \int \mathrm{d} \mathbf{r}^{N_{s}} \exp \left(-U\left[x^{\prime}{ }_{i}+\alpha a z_{i} / L_{z}, y_{i}, z_{i}\right] / k_{\mathrm{B}} T\right)
$$

Its differentiation with respect to $\alpha a$ is written as

$$
\frac{\partial Z_{N_{\mathrm{f}}}}{\partial \alpha a}=-\frac{1}{k_{\mathrm{B}} T L_{z}} \int \mathrm{d} \mathbf{r}^{N_{\mathrm{f}}} \int \mathrm{d} \mathbf{r}^{N_{\mathrm{s}}} \exp \left(-U / k_{\mathrm{B}} T\right) \frac{\partial U}{\partial \alpha a}
$$

Finally, we can obtain $T_{z x}$ of the fully atomistic system as follows: 


$$
\begin{aligned}
T_{z x}= & \frac{1}{2 A} \sum_{i=1}^{N_{f}} \sum_{j \neq i}^{N_{f}}<u^{\prime}{ }_{\mathrm{ff}}\left(r_{i j}\right) x_{i j} z_{i j} /\left(r_{i j} L_{z}\right)> \\
& +\frac{1}{A} \sum_{i=1}^{N_{f}} \sum_{j=1}^{N_{\mathrm{s}}}<{u^{\prime}}_{\mathrm{fs}}\left(r_{i j}\right) x_{i j} z_{i j} /\left(r_{i j} L_{z}\right)> \\
& +\frac{1}{2 A} \sum_{i=1}^{N_{s}} \sum_{j=1}^{N_{s}}<u_{\mathrm{ss}}^{\prime}\left(r_{i j}\right) x_{i j} z_{i j} /\left(r_{i j} L_{z}\right)> \\
& +\frac{1}{A} \sum_{i=1}^{N_{f}} \sum_{j=1}^{N_{\mathrm{w}}}<u^{\prime}{ }_{\mathrm{fs}}\left(r_{i j}\right) x_{i j} z_{i j} /\left(r_{i j} L_{z}\right)> \\
& +\frac{1}{A} \sum_{i=1}^{N_{\mathrm{s}}} \sum_{j=1}^{N_{\mathrm{w}}}<u_{\mathrm{ss}}^{\prime}\left(r_{i j}\right) x_{i j} z_{i j} /\left(r_{i j} L_{z}\right)> \\
& +\frac{1}{A} \sum_{i=1}^{N_{\mathrm{w}}^{t}} \sum_{j=1}^{N_{\mathrm{w}}^{\mathrm{b}}}<u_{\mathrm{ss}}^{\prime}\left(r_{i j}\right) x_{i j} z_{i j} /\left(r_{i j} L_{z}\right)>
\end{aligned}
$$

where $u_{\mathrm{ab}}^{\prime}=\frac{\partial u_{\mathrm{ab}}}{\partial r_{i j}}=-24 \varepsilon_{\mathrm{ab}}\left(2 \sigma^{12} / r_{i j}^{13}-\sigma^{6} / r_{i j}^{7}\right)$. The angular brackets $\langle W\rangle$ signify the ensemble average

$$
<W>=\sum_{L_{z}} \int \mathrm{d} \mathbf{r}^{N_{f}} \int \mathrm{d} \mathbf{r}^{N_{s}} P\left(\mathbf{r}^{N_{f}}, \mathbf{r}^{N_{s}} ; L_{z}\right) W\left(\mathbf{r}^{N_{f}}, \mathbf{r}^{N_{s}} ; L_{z}\right)
$$

where $P$ is the isothermal-isobaric distribution function.

For the hybrid system, by carrying out the integrals over the formal momentum space $\left(\mathbf{p}^{N_{\mathrm{f}}}, \mathbf{p}^{N_{s}^{\prime}}, \mathbf{P}^{N_{\mathrm{n}}}\right)$, we can obtain the canonical partition function $Q_{N_{\mathrm{f}}, \mathrm{c}}$ which can be expressed as a classical limit as

$$
\begin{gathered}
Q_{N_{f}, \mathrm{c}}=\frac{1}{h^{3\left(N_{\mathrm{f}}+N^{\prime} \mathrm{s}+N_{\mathrm{n}}\right)}} \frac{1}{N_{\mathrm{f}} !} \int \mathrm{d} \mathbf{p}^{N_{\mathrm{f}}} \int \mathrm{d} \mathbf{r}^{N_{\mathrm{f}}} \int \mathrm{d} \mathbf{p}^{N^{\prime} s} \int \mathrm{d} \mathbf{r}^{N^{\prime} s} \int \mathrm{d} \mathbf{P}^{N_{\mathrm{n}}} \\
\int \mathrm{d} \mathbf{R}^{N_{\mathrm{n}}} \exp \left(-\tilde{H}_{\mathrm{c}} / k_{\mathrm{B}} T\right)
\end{gathered}
$$

where

$$
\begin{aligned}
\tilde{H}_{\mathrm{c}}= & \sum_{i=1}^{N_{f}} \mathbf{p}_{i}^{2} / 2 m_{\mathrm{f}}+\sum_{i=1}^{N^{\prime} \mathrm{s}} \mathbf{p}_{i}^{2} / 2 m_{\mathrm{s}}+\frac{1}{2} \mathbf{P}^{\mathrm{T}} \mathbf{M}^{-1} \mathbf{P} \\
& +U_{\mathrm{c}}\left(\mathbf{r}^{N_{\mathrm{f}}}, \mathbf{r}^{N_{\mathrm{s}}}, \mathbf{R}^{N_{\mathrm{n}}}\right)
\end{aligned}
$$

$\mathbf{P}$ is the $3 N_{n}$-dimensional column vector of the momenta conjugate to the nodal coordinates $\mathbf{R}^{N_{n}}$ and $\mathbf{M}^{-1}$, which is the effective mass matrix and it depends on the transformation in Eq. (6). After the integral on the momenta, Eq. (26) can be rewritten as

$$
Q_{N_{\mathrm{f}}, \mathrm{c}}=\frac{1}{h^{3 N_{\mathrm{n}}}} \frac{1}{N_{\mathrm{f}} !} \Lambda_{\mathrm{f}}^{3 N_{\mathrm{f}}} \Lambda_{\mathrm{s}}^{3 N^{\prime} \mathrm{s}} \int \mathrm{d} \mathbf{P}^{N_{\mathrm{n}}} \exp \left(-\mathbf{P}^{\mathrm{T}} \mathbf{M}^{-1} \mathbf{P} / 2 k_{\mathrm{B}} T\right) Z_{N_{\mathrm{f}}, \mathrm{c}}
$$

where

$$
Z_{N_{\mathrm{f}}, \mathrm{c}}=\int \mathrm{d} \mathbf{r}^{N_{\mathrm{f}}} \int \mathrm{d} \mathbf{r}^{N^{\prime} s} \int \mathrm{d} \mathbf{R}^{N_{\mathrm{n}}} \exp \left(-U_{\mathrm{c}} / k_{\mathrm{B}} T\right)
$$

By performing a similar derivation, we obtained the shear stress $T_{z x, c}$ of the hybrid system as follows:

$$
\begin{aligned}
& T_{z x, \mathrm{c}}=\frac{1}{2 A} \sum_{i=1}^{N_{f}} \sum_{j \neq i}^{N_{f}}<u_{\text {fff }}^{\prime}\left(r_{i j}\right) x_{i j} z_{i j} /\left(r_{i j} L_{z}\right)> \\
& +\frac{1}{A} \sum_{i=1}^{N_{f}} \sum_{j=1}^{N_{\mathrm{w}}^{\mathrm{b}}}<u^{\prime}{ }_{\mathrm{fs}}\left(r_{i j}\right) x_{i j} z_{i j} /\left(r_{i j} L_{z}\right)> \\
& +\frac{1}{A} \sum_{i=1}^{N_{f}} \sum_{j=1}^{N^{\prime} s}<u^{\prime}{ }_{\mathrm{fs}}\left(r_{i j}\right) x_{i j} z_{i j} /\left(r_{i j} L_{z}\right)> \\
& +\frac{1}{2 A} \sum_{i=1}^{N^{\prime} s} \sum_{j \neq i}^{N^{\prime} s}<u_{\mathrm{ss}}^{\prime}\left(r_{i j}\right) x_{i j} z_{i j} /\left(r_{i j} L_{z}\right)> \\
& +\frac{1}{2 A} \sum_{i=1}^{N^{\prime} s} \sum_{j=1}^{N^{\prime \prime s}}\left\langle u_{\mathrm{ss}}^{\prime}\left(r_{i j}\right) x_{i j} z_{i j} /\left(r_{i j} L_{z}\right)>\right. \\
& +\frac{1}{A} \sum_{i=1}^{N^{\prime} s} \sum_{j=1}^{N_{\mathrm{w}}^{\mathrm{b}}}\left\langle u_{\mathrm{ss}}^{\prime}\left(r_{i j}\right) x_{i j} z_{i j} /\left(r_{i j} L_{z}\right)>\right. \\
& +\frac{1}{2 A} \sum_{i=1}^{N_{\mathrm{w}}^{t}} \sum_{j=1}^{N^{\prime \prime}}<u_{\mathrm{ss}}^{\prime}\left(r_{i j}\right) x_{i j} z_{i j} /\left(r_{i j} L_{z}\right)> \\
& +\frac{1}{A} \sum_{\mathrm{e}=1}^{N_{\mathrm{e}}} N_{\mathrm{a}}^{\mathrm{e}}\left[\frac{1}{2} \sum_{j \neq i}\left\langle u_{\mathrm{ss}}^{\prime}\left(r_{i j}\right) x_{i j} z_{i j} /\left(r_{i j} L_{z}\right)>\right]\right. \\
& +\frac{1}{A} \sum_{\mathrm{e}=1}^{N_{\mathrm{e}}} N_{q}^{\mathrm{e}}\left[\frac{k_{\mathrm{B}} T}{2}<\frac{1}{|D|} \frac{\partial|D|}{\partial \alpha a}>\right]
\end{aligned}
$$

\section{Computational procedure}

We surveyed the behaviors of the system under reversible shearing for a given atomistic number $N_{\mathrm{f}}+N_{\mathrm{s}}$ or $N_{\mathrm{f}}+N^{\prime}{ }_{\mathrm{s}}+N_{\mathrm{n}}$, the temperature $T$, and the normal stress $-T_{z z}$. We also investigated the effects of the substrate's elasticity, temperature, and filmsubstrate coupling strength on the shear stress. To compute the thermomechanical properties, we performed isothermal-isobaric Monte Carlo (MC) simulations [33]. The shearing process, which is considered to be quasistatic (reversible), is performed by increasing $\alpha$ gradually in small increments by $\Delta \alpha$. 
For the register $\alpha=0$, the initial film atoms are randomly placed between the two solid surfaces. The initial configuration of the system for the register $\alpha \neq 0$ is taken from the last configuration of the previous register $\alpha-\Delta \alpha$, which is combined with a linear increment $(0 \leq \Delta x(z) \leq \Delta \alpha)$ of the several rows near the top wall. One MC cycle consists of diffusive steps of atoms and nodes in which $L_{z}$ is fixed or exchanged. To facilitate the equilibration of the fluid film, the fluid atoms are subjected to a normal move cycle and several large move cycles. We generated a total of $M$ MC cycles; the first half of these cycles were discarded and we evaluated the ensemble averages for the remaining half. At some transition points from the positive shear stresses to the negative ones or vice versa, we extended the total number of $\mathrm{MC}$ cycles to $2 \mathrm{M}$ in order to reach the quasistatic states of the system.

For the simulations, Table 1 lists the values of the various parameters for the fully atomistic and multi-scale simulations. The numerical values are expressed as dimensionless units based on the LennardJones parameters for the solid-solid interaction. The units of these parameters are expressed as follows: the distance is $\sigma$, the energy is $\varepsilon_{\mathrm{ss}}$, the stress is $\varepsilon_{\mathrm{ss}} / \sigma$, and the temperature is $\varepsilon_{\mathrm{ss}} / k_{\mathrm{B}}$.

Table 1 Parameters of the fully atomic and multi-scale simulations of the thin-film lubrication.

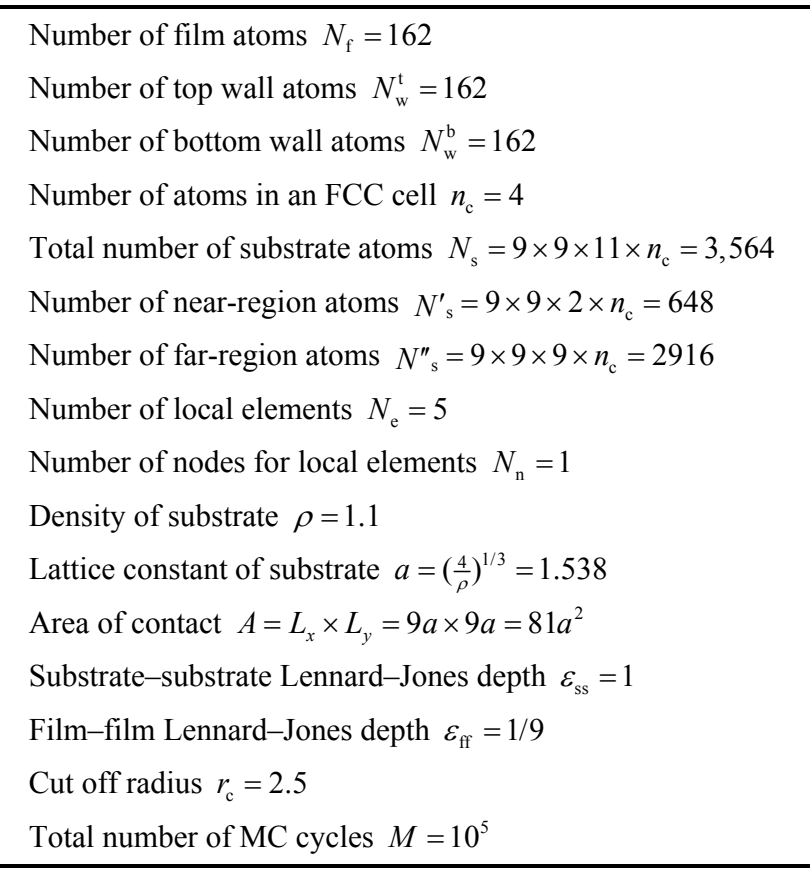

\section{Results}

\subsection{Fully atomistic structure of the substrate}

This study first investigates the tribological behavior of the fully atomistic version of the thin-film lubrication system as shown in Fig. 1(a). Figure 3 displays the shear stress $T_{z x}$ and the mean separation $L_{z}$ as a function of the register $\alpha$ at $\varepsilon_{\mathrm{fs}}=1 / 9, T=0.15$, and $T_{z z}=-1.0$. It is noted that the forward and backward processes in $\alpha$ are periodic with a length of 1 . The forward process composites two elastic (stick) zones $(0 \leq \alpha \leq 0.6,0.7 \leq \alpha \leq 1)$ and a transition (slip) zone $(0.6 \leq \alpha \leq 0.7)$. At $\alpha=0$, the fluid atoms converge to a stable FCC crystal structure with solid atoms of the substrate and the bottom wall. The perfect symmetric atomistic structure of the system in the equilibrium state leads to $T_{z x}=0$. As $\alpha$ increases gradually in the range $0<\alpha \leq 0.6$, the substrate connected with the
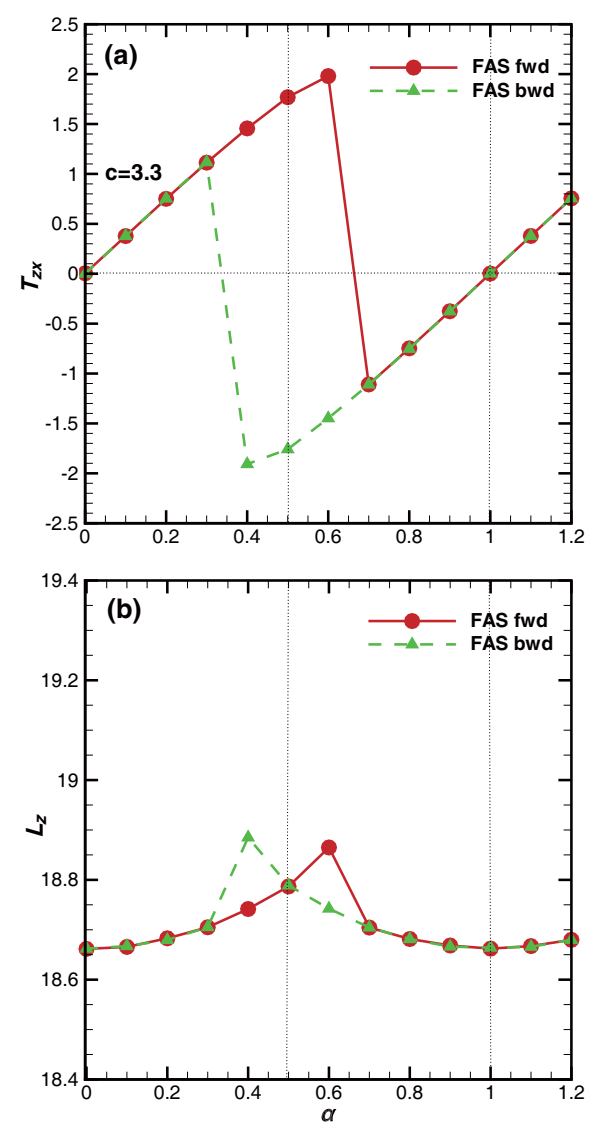

Fig. 3 Shear stress profile $\left(T_{z x}\right.$ vs register $\left.\alpha\right)$ and the mean separation profile ( $L_{z}$ vs. register $\alpha$ ) of the thin-film lubrication system with $\varepsilon_{\mathrm{fs}}=1 / 9$ at $T=0.15$ and $T_{z z}=-1.0$ (forward, a solid line with circles; backward, a dashed line with triangles). 
bottom wall through the film similar to a whole crystal generates an elastic shear behavior in the $x$ direction. Even when $\alpha=0.5$, the substrate presenting the elastic shear behavior postpones the appearance of the transition process in the system. As illustrated in Fig. 4(a), although the solid atoms in the two surfaces and the fluid atoms in the thin film are still matched to the FCC crystal structure, the solid atoms in the substrate have the right inclination to those in the bottom wall. The fluid atoms in the film are thus confined in the interstices. A fluid atom in its interstice is closer to the upper solid atom, which lies to its left and the lower one lies to its right. A reversed shear stress (in the negative $x$ direction) on the upper surface is generated by the thin fluid film, transmitted to the top wall, and is counterbalanced by the external stress that is holding the top wall in the shifting place. Meanwhile, as the shear stress in the film increases, the interstices are compressed so it is more slender, and then the effective film thickness increases along with the mean separation $L_{z}$. As $\alpha$ varies in the range of $0.6 \leq \alpha \leq 0.7$, the shear stress and the effective

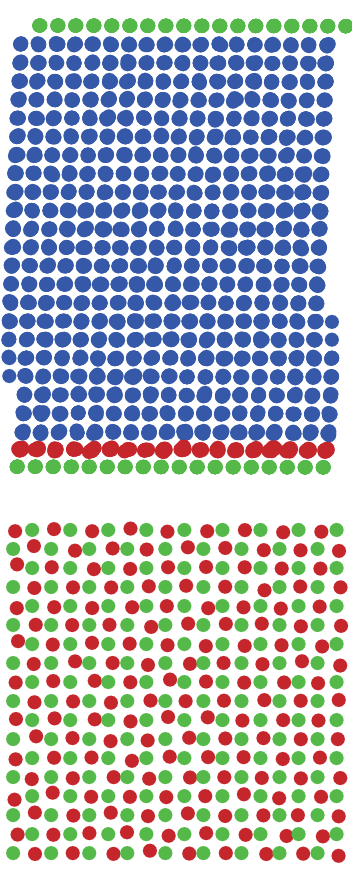

(a)
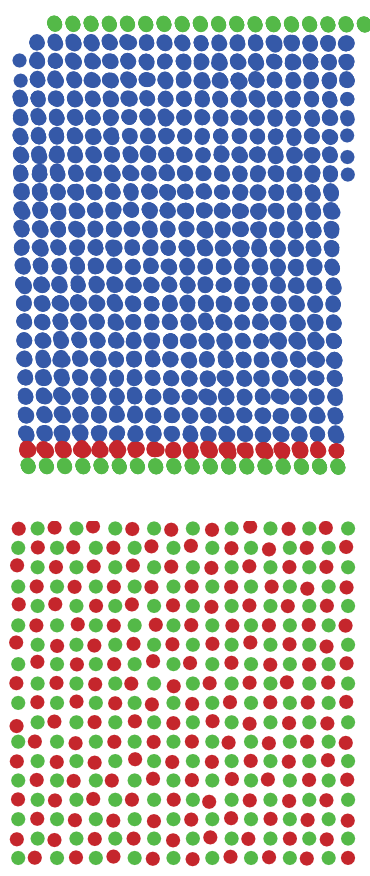

(b)
Fig. 4 Atomistic structures $(x-z$ side view and $x-y$ bottom view) of the tribological system at $\alpha=$ (a) 0.6 and (b) 0.7 . The wall, the elastic substrate, and the fluid atoms are represented by the green, blue, and red spheres, respectively. film thickness reaches its largest values. The atoms in the film or in the upper surface of the substrate abruptly step over the atoms that contact them. To complete the relative striding of the atoms in the system, the substrate must have sharp climbing to provide the vertical space near the film. Since the slip zone is very small, the atoms in the thin film are almost regular and they cannot rejoin the fluidic state. When $\alpha$ increases further in the range of $0.7 \leq \alpha \leq 1$, the systematic shear stress curve has a central rotational symmetry of $1-\alpha$. An elastic shear behavior is generated in the substrate that is connected to the bottom wall through the film. As demonstrated in Fig. 4(b), the solid atoms in the two surfaces and the fluid atoms in the thin film are matched to a new FCC crystal structure. The solid atoms in the substrate have the left inclination to those in the bottom wall; hence, the fluid atoms in the film are confined in the interstices. A fluid atom in its interstice is closer to the upper solid atom, which lies to its right and the lower one lies to its left. A shear stress (in the $x$ direction) on the upper surface is generated by the thin fluid film, transmitted to the top wall, and it is counterbalanced by the external stress that holds the top wall in the shifting place. Meanwhile, as the shear stress in the film decreases, the interstices are changed into stubbier, and then the effective film thickness decreases along with the mean separation $L_{z}$. When the register finally reaches $\alpha=1$, the system returns to the same state when $\alpha=0$. The solid atoms in the substrate have a relative sliding distance $a$ to those in the bottom wall. As $\alpha$ increases from 1 to 1.2, the above profiles in the range $(0<\alpha \leq 0.2)$ are repeated. In the reversed slip process, shearing from $\alpha=1.2$ to $\alpha=0$ presents the same stick-slip behavior as the shearing in the forward direction. The shear stress profile has central rotational symmetry with the forward process. In the zone away from the transition process, the shearing carries the system in reverse in the same states as the shearing in the forward direction. However, the states near the slip zone in the backward process do not coincide with those in the forward process, which forms a hysteresis loop. As a result, the fluid atoms in the film are confined in the slender interstices that are composed of the upper-left and lower-right solid atoms in the surfaces before the 
slipping transition of the substrate. The hysteresis phenomenon reflects that the samples of the configurational space can reach the equilibrium state and complete the slipping transition, which appear at a low frequency. The ensemble average can only be based on a biased configurational distribution rather than the Boltzmann distribution. Theoretically, increasing the number of the MC cycles should reduce the size of the hysteresis loop [34]. The configuration of the system is almost stuck, even though the number of MC cycles is large enough. Therefore, the system tends to have a constrained equilibrium and it preserves the hysteresis. The dependence of the hysteresis on the elasticity of the substrate, the temperature, and the load of the system will be mentioned in the following subsections.

\subsection{Effects of the elastic substrate and its appro- ximate treatment}

To determine the importance of properly accounting for the elasticity of the substrate in the thin-film lubrication system, this study took four modeling tribological systems. These were the successively enlarged elastic region of the substrate with a 0-layer near region (wholly rigid substrate or the crystalline model) that is presented in Fig. 1(c), the 8-layer near region (partly rigid substrate) displayed in Fig. 1(d), the 16-layer near region (partly rigid substrate), and the 22-layer near regions (fully atomistic substrate). In the systems, except for the fixed $N_{\mathrm{f}}, N_{\mathrm{w}}^{\mathrm{t}}$, and $N_{\mathrm{w}}^{\mathrm{b}}$ are provided in Table $1, N_{s}$ is changed to $162 l$, where $l=0,8,16,22$. The shear stress profiles of the systems at $\varepsilon_{\mathrm{fs}}=1 / 9, T=0.15$, and $T_{z z}=-1.0$ are depicted in Fig. 5.

For the tribological system with a wholly rigid substrate, the shear stress of the system cannot be relaxed in the rigid substrate and it directly acts on the top wall. The thermal elastic coefficient reaches a large value $\left(c_{0}=\Delta T_{z x} / \Delta \alpha=8.8\right)$, which reveals that the system loses its elastic effects on the substrate. The process of slipping over the atoms in the film or in the bottom wall appears early. The stick zone $(0 \leq \alpha \leq 0.2,0.8 \leq \alpha \leq 1)$ in the forward process is narrow, but the slip zone $(0.2 \leq \alpha \leq 0.8)$ is wide. In the stick zone, as demonstrated in Figs. 6(a) and 6(c), the solid atoms in the top wall have the right and the

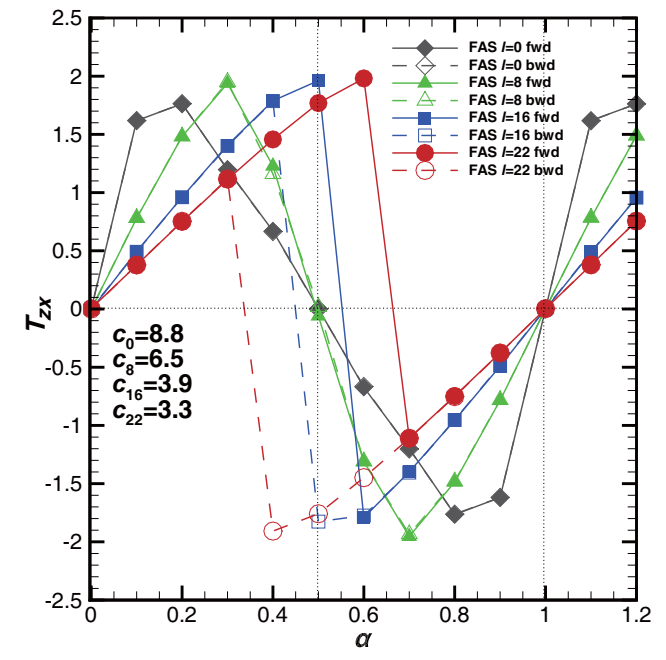

Fig. 5 Shear-stress profile ( $T_{z x}$ versus register $\alpha$ ) for the models with the elastic substrates with a different thickness $l$ at $\varepsilon_{\mathrm{fs}}=1 / 9$, $T=0.15$, and $T_{z z}=-1.0$ (forward, with a solid line; backward, with a dashed line). Wholly rigid substrate/0-layer near region (diamonds); 8-layer near region (triangles); 16-layer near region (squares); and fully atomistic/22-layer near region (circles).

left, respectively, inclination to those in the bottom wall. The fluid atoms in the film are confined in the interstices. The solid atoms in the two surfaces and the fluid atoms in the film are matched to the FCC crystal structure. In the slip zone, as illustrated in Fig. 6(b), the film atoms between the two surfaces are irregular and they rejoin the fluidic state. This transition state does not appear in the fully atomistic version of the tribological system. Meanwhile, shearing from $\alpha=1.2$ to $\alpha=0$ carries the system in reverse through precisely the same states as shearing in the forward direction. The hysteresis loop in the fully atomistic version of the tribological system vanishes in the system due to the inexistence of the elastic substrate.

For the tribological system with partly rigid substrates, the thermal elastic coefficient $\left(c_{l}=\Delta T_{z x} / \Delta \alpha\right)$ decreases gradually by increasing the thickness $l$ for the elastic region of the substrate. The largest shear stress $T_{z x}^{*}$ has a slight increase in comparison to the system where $l=0$ and it remains constant when $l \geq 8$. The stick zones are enlarged and the thermal elastic constants $c_{l}$ decreases. The slip zones are narrowed; hence, the transition process from the positive $T_{z x}^{*}$ to the negative one becomes quicker. However, the shear-stress profile and atomistic structures depend on the elastic region's thickness $l$ of the substrate. For the tribological system with an 8-layer near region, the shear-stress profile 


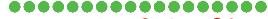
0.00000000000 (6)

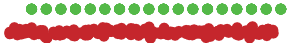

ceecececeecect

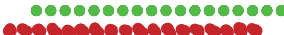

cocesceces (1)

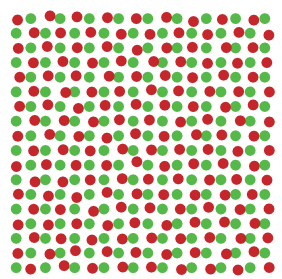

(a)

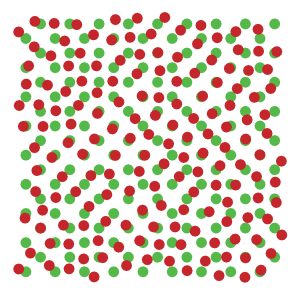

(b)

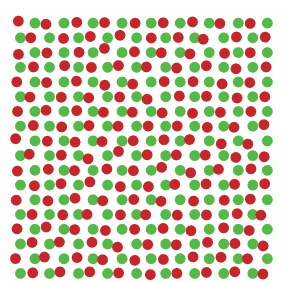

(c)

Fig. 6 Atomistic structures ( $x-z$ side view and $x-y$ bottom view) of the modeling tribological system at $\alpha=$ (a) 0.2 , (b) 0.5 , and (c) 0.8 . The wall, the rigid substrate, and the fluid atoms are represented by the green, green, and red spheres, respectively.

and atomistic structures possess similar behaviors with those for the tribological system with a wholly rigid substrate. The stick-slip behavior in the forward process is performed reversibly and it coincides with the backward process. For the tribological system with a 16-layer near region, the shear-stress profile and atomistic structures are similar to those for the fully atomistic version of the tribological system. The states away from the slip zone in the forward process coincide with those in the backward process, but the states near the slip zone do not coincide with those in the forward process. This leads to a hysteresis loop in the forward and backward processes.

Therefore, the approximate treatment (wholly $(l=0)$ or partly rigid $(l=8))$ of the substrate in the thin-film lubrication system can only predict the approximate and partial tribological features in the fully atomistic model. The elasticity of the substrate has a marked impact on the appearance of the hysteresis loop. To generate the qualitative tribological behavior with a hysteresis loop in the fully atomistic model, the elastic substrate of the thin-film lubrication system must have an adequate thickness of $l \geq 16$. In comparison to the tribological system with the wholly rigid substrate, the hysteresis loop is almost a signature of the elastic response of the substrate in a fully atomistic version of the thin-film lubrication system.

\subsection{Hybrid treatment of the substrate}

As depicted in Fig. 1(b), the substrate of the tribological system is treated as a hybrid description with the local elements. The parameters for the multi-scale simulation are given in Table 1. By using the multi-scale method, the shear stress $T_{z x, \mathrm{c}}$ and the mean separation $L_{z, \mathrm{c}}$ of the system at $\varepsilon_{\mathrm{fs}}=1 / 9, T=0.15$, and $T_{z z}=-1.0$ are determined and are compared with those that are obtained from the fully atomistic simulation in Fig. 7. In addition, $T_{z x, \mathrm{c}}$ and $L_{z, \mathrm{c}}$ equal the "exact" (fully atomistic) values in the forward and backward processes. The stick-slip phenomenon and the corresponding physical mechanism based on the atomistic structures coincide with those in the fully atomistic version of the tribological system. This reveals that the elastic response of the substrate can be effectively accounted for in the hybrid scheme. Meanwhile, the computing time of the multi-scale simulation based on the hybrid scheme is only about $1 / 5$ of the fully atomistic simulation. In comparison to the fully atomistic simulation, the multi-scale simulation based on the hybrid scheme can significantly reduce the computing time and increase the computational efficiency.

To confirm the validity of the multi-scale simulation of the thin-film lubrication system further, the temperature $T$ and the film-substrate coupling strength $\varepsilon_{\mathrm{fs}}$ are chosen as the variable parameters of the system in the following subsections.

\subsubsection{Thermal effects}

Figure 8 displays the shear stress profiles of the system for a few temperatures ( $T=0.05,0.15$, and 0.25$)$, which 

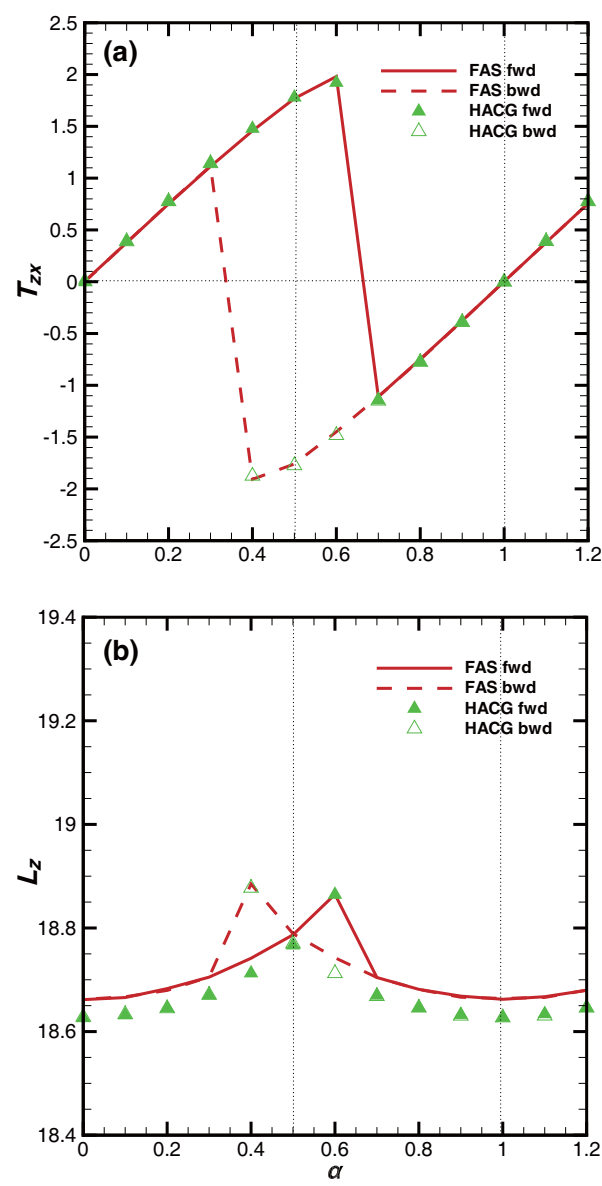

Fig. 7 Shear stress profiles at $\varepsilon_{\mathrm{fs}}=1 / 9, T=0.15$, and $T_{z z}=-1.0$ are generated in the fully atomistic and multi-scale simulations. The fully atomistic profile (forward, with a solid line; backward, with a dashed line) and multi-scale profile (forward, with filled triangles; backward, with hollow triangles).

was fixed at $\varepsilon_{\mathrm{fs}}=1 / 9$ and $T_{z z}=-1.0 . T_{z x, \mathrm{c}}$ generated by the multi-scale simulation is also in excellent agreement with $T_{z x}$ by the fully atomistic simulation. The thermal elastic coefficient $c$ decreases as the temperature increases. This reveals that the stiffness of the tribological system is reduced; hence, the largest shear stress $T_{z x}^{*}$ decreases and the transition process appears earlier. Meanwhile, the hysteresis loops in the forward and backward processes are smaller due to the decreasing/increasing of the stiffness/elasticity of the substrate.

\subsubsection{Effects of the film-substrate coupling strength}

Figure 9 displays the shear stress profiles of the system for the film-substrate coupling strengths of $\varepsilon_{\mathrm{sf}}=1 / 3$, $1 / 9$, and $1 / 12$, and they are fixed at $T=0.15$ and $T_{z z}=-1.0 . T_{z x, \mathrm{c}}$, which is generated by the multi-scale
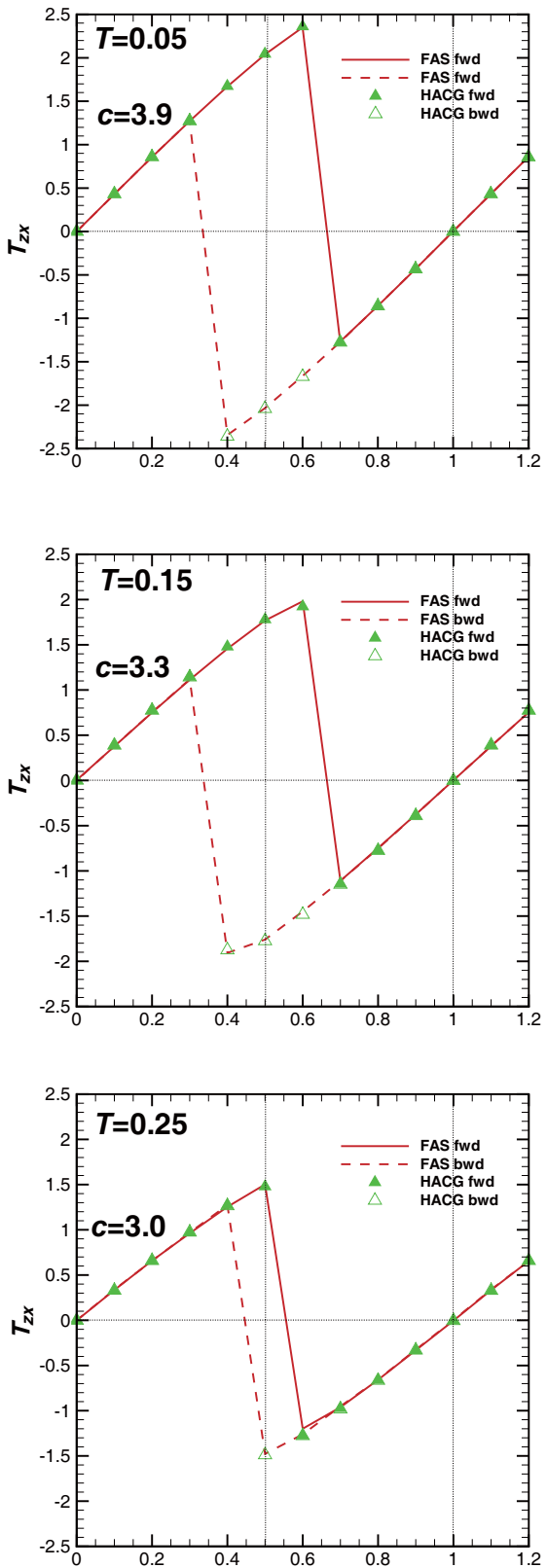

Fig. 8 Shear stress profiles at $\varepsilon_{\mathrm{fs}}=1 / 9, T_{z z}=-1.0$, and (a) $T=0.05$, (b) $T=0.15$, and (c) $T=0.25$ are generated in the fully atomistic and multi-scale simulations. The fully atomistic profile (forward, with a solid line; backward, with a dashed line) and multi-scale profile (forward, with filled triangles; backward, with hollow triangles).

simulation, is also in excellent agreement with $T_{z x}$ by the fully atomistic simulation. The thermal elastic coefficients $c$ are almost constant in the process of changing the film-substrate coupling strength $\varepsilon_{\text {sf }}$. This indicates that in the tribological system, the intermolecular interaction between the substrate and the bottom wall is dominant in comparison to the intermolecular interaction between the film and the 

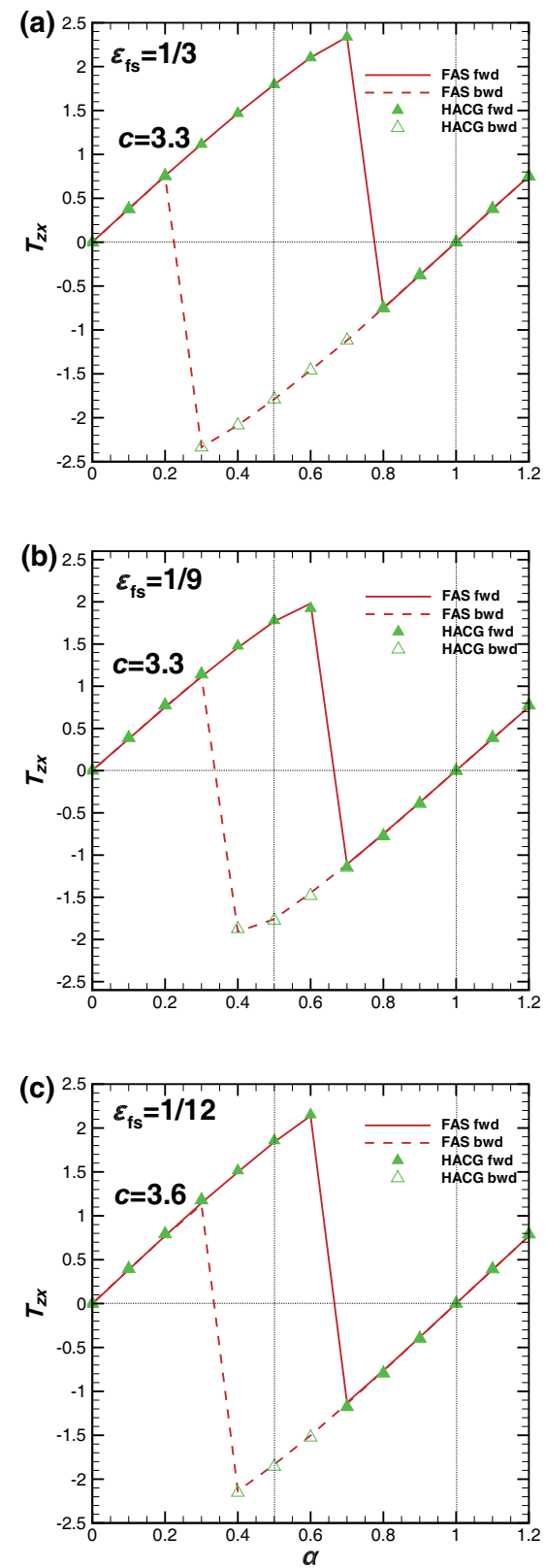

Fig. 9 Shear stress profiles at $T=0.15, T_{z z}=-1.0$, and (a) $\varepsilon_{\mathrm{fs}}=1 / 3$, (b) $\varepsilon_{\mathrm{fs}}=1 / 9$, and (c) $\varepsilon_{\mathrm{fs}}=1 / 12$ are generated in the fully atomistic and multi-scale simulations. The fully atomistic profile (forward, with a solid line; backward, with a dashed line) and multi-scale profile (forward, with filled triangles; backward, with hollow triangles).

substrate (or the bottom wall). The film-substrate coupling strength, which describes the intermolecular interaction between the thin film and the substrate (or the bottom wall), hardly affects the stiffness of the tribological system. For the film-substrate coupling strength $\varepsilon_{\mathrm{sf}}=1 / 3, \varepsilon_{\mathrm{sf}}$ is equal to $\varepsilon_{\mathrm{ss}}$, i.e., the intermolecular interaction between the film and the substrate is the same with the substrate atoms. This directly leads to the increase in the adhesive force of the fluid atoms to the solid surfaces and the delaying of the slip process. A large hysteresis loop appears in the forward and backward processes. For the filmsubstrate coupling strength $\varepsilon_{\mathrm{sf}}=1 / 12, \varepsilon_{\mathrm{sf}}$ is smaller than $\varepsilon_{\mathrm{ss}}$. The tribological system has a weaker adhesive force than $\varepsilon_{\mathrm{sf}}=1 / 9$. As a result, both systems with $\varepsilon_{\mathrm{sf}}=1 / 9$ and $1 / 12$ have similar tribological behaviors.

From the results above, we can conclude that the multi-scale method is effective to predict the tribological behavior of the thin-film lubrication systems in the range of the parameters (i.e., temperature and filmsubstrate coupling strength).

\subsection{Application of the multi-scale method}

As an application of the multi-scale method, this study examined three 3D tribological systems with mono-layer, two-layer, and three-layer molecular thin-films to investigate the tribological behavior. Schematics of the hybrid atomistic and coarse-grained treatment of the two-layer and three-layer molecular thin-film lubrication systems are presented in Fig. 10. Using the multi-scale method, this study focused on the effects of the loads on the tribological behaviors of the systems under reversible shearing for a given atomistic number $n_{l} N_{\mathrm{f}}+N^{\prime}{ }_{\mathrm{s}}+N_{\mathrm{n}}$, temperature $T=$ 0.15 , and load $-T_{z z}$, where $n_{l}=1,2$, and 3 , which corresponds to the mono-layer, two-layer, and threelayer films, respectively. The film-substrate coupling strength is fixed as $\varepsilon_{\mathrm{sf}}=1 / 9$.

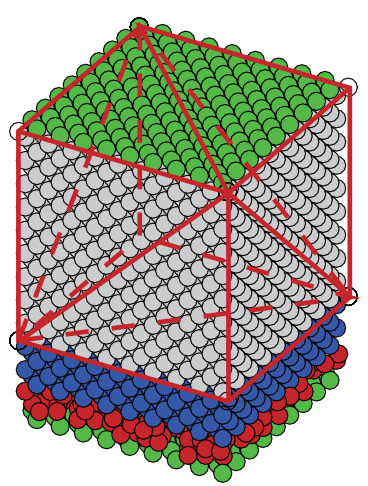

(a)

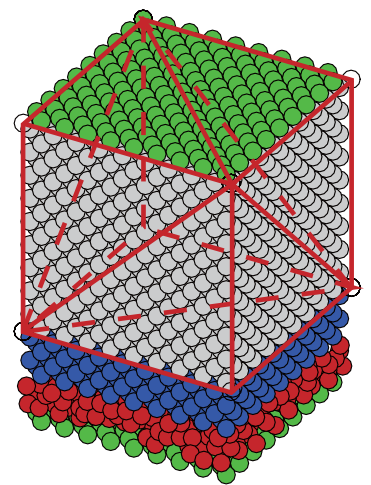

(b)
Fig. 10 Schematic diagram of three-dimensional (a) two-layer and (b) three-layer molecular thin-film lubrication system with periodic boundary conditions in the $x$ - and $y$-directions. The wall, the elastic substrate, and the fluid atoms are represented by the green, blue, and red spheres, respectively. The coarse-grained far region of the substrate is represented by the gray spheres. 
Figure 11(a) presents the shear stress $T_{x z}$ vs. the $\alpha$ curves under several loads $-T_{z z}$ for the mono-layer molecular thin-film. The stick-slip processes with the hysteresis loops appear when the load is $-T_{z z} \geq 0.5$.
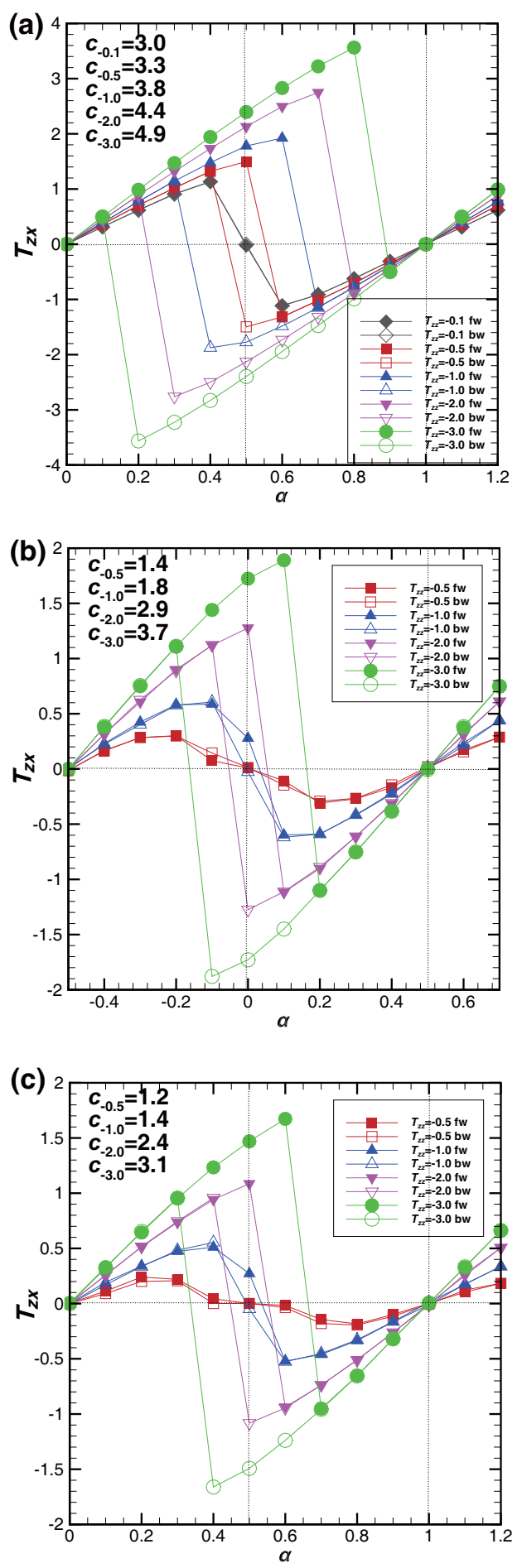

Fig. 11 Shear stress $T_{x z}$ vs. the $\alpha$ curves under several loads $-T_{z z}$ for (a) the mono-layer, (b) two-layer, and (c) the three-layer molecular thin-film.
At the lightest load $-T_{z z}=0.1$, the curve in the forward process coincides with the backward process. The molecular tribological mechanism that is based on the stick-slip processes was previously analyzed in Section 4.1, where the load $-T_{z z}=1.0$ is considered as an example. The thermal elastic coefficient $c_{T z z}$ increases monotonously as the load $-T_{z z}$ increases, which leads to a larger shear stress and a hysteresis loop in the forward and backward processes. The heavier the load, the more compressed the confined film. This generates a stronger intermolecular force to prevent sliding between the two solid surfaces.

Figures 11(b) presents the shear stress $T_{x z}$ vs. the $\alpha$ curves under several loads $-T_{z z}$ for the twolayer molecular thin-film. In comparison with the mono-layer film tribological model, on one hand, the equilibrium state with a stable FCC crystal structure between the thin-film and the two surfaces is changed from $\alpha=0 / 1$ to $-0.5 / 0.5$; consequently, the stick-slip period in the $T_{x z}-\alpha$ profile is changed from $[0,1]$ to $[-0.5,0.5]$. The stick-slip process with a hysteresis loop appears when the load is $-T_{z z} \geq 2.0$. For the lighter loads where $-T_{z z}=0.5$ and 1.0, the curves in the forward processes coincide with those in the backward processes. As shown in Figs. 12(a) and 12(c), since the fluid atoms (open red spheres) in the secondlayer film serve as the solid atoms to form the stable FCC crystal structures, the solid atoms in the substrate at $\alpha=-0.2$ and 0.2 have the right and left inclination to those in the bottom wall, respectively. The fluid atoms are confined in the interstices. A transitional state appears at $\alpha=0$ and it postpones the slipping process. The fluid atoms (filled and open red spheres) in the two-layer thin-film are placed irregularly between the two surfaces as demonstrated in Fig. 12(b). On the other hand, the thermal elastic coefficient $c_{T z z}$ increases monotonously with the load $-T_{z z}$ for the same reason that the shear stress and the hysteresis loop increases with the load. The difference is that $c_{T z z}$ and $T_{x z}$ for a fixed $-T_{z z}$ are markedly reduced from the mono-layer film system to the two-layer film system. The thicker the confined film, the weaker the intermolecular force between the two solid surfaces. This can promote the sliding between the two surfaces. 

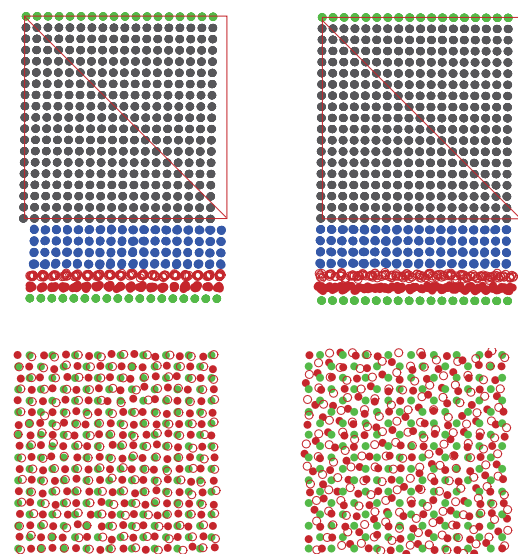

(a)

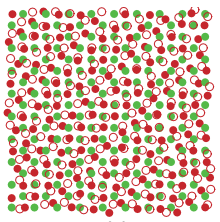

(b)
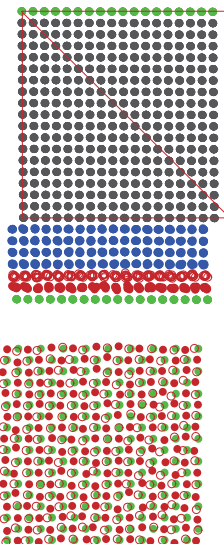

(c)
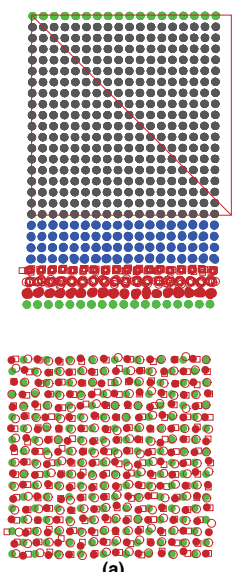

(a)
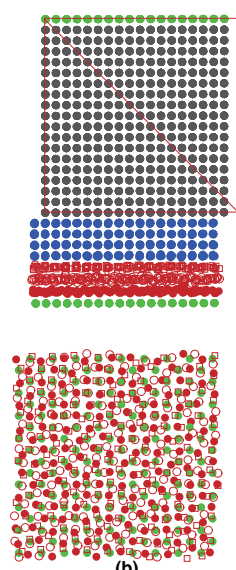

(b)
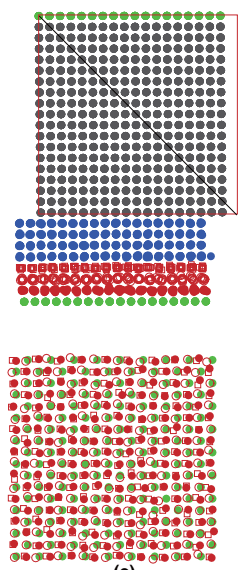

(c)

Fig. 12 Atomistic structures ( $x-z$ side view and $x-y$ bottom view) of the two-layer molecular tribological system at (a) $\alpha=-0.2$, (b) $\alpha=0.0$, and (c) $\alpha=0.2$. The fluid atoms in the second layer are represented by the open red spheres. The wall, the elastic substrate, and the fluid atoms in the first layer are represented by the green, blue, and filled red spheres, respectively. The coarse-grained far region of the substrate is represented by the gray spheres.

Figure 11(c) presents the shear stress $T_{x z}$ versus the $\alpha$ curves under several loads $-T_{z z}$ for the three-layer molecularly thin-film. In comparison to the two-layer film tribological model, the equilibrium state and the stick-slip period return to the mono-layer film system. As shown in Figs. 13(a) and 13(c), the second-layer fluid atoms (open red spheres) and the third-layer fluid atoms (open read squares) in the thin-film at $\alpha=0.3$ and 0.7 serve as the solid atoms that form the stable FCC crystal structures. The fluid atoms (filled and open red spheres, and open squares) in the three-layer thin-film at $\alpha=0.5$ are placed irregularly between the two surfaces as illustrated in Fig. 13(b). Most tribological behaviors resemble the two-layer film system. The difference is that the thermal elastic coefficient $c_{T z z}$ and the shear stress $T_{x z}$ at a fixed load are slightly reduced from the two-layer film system to the three-layer film system. This reveals that the three-layer film that replaces the two-layer film can only slightly reduce the intermolecular force between the two solid surfaces and promote the sliding between them. For the more-layer film system, the intermolecular force in the fluid film should be dominant to govern the sliding process in comparison to the intermolecular force between the two solid surfaces. It was determined [9] that for the $n_{l}(\geq 4)$-layer thin film system, the equivalent viscosity of the film increases as the film

Fig. 13 Atomistic structures ( $x-z$ side view and $x-y$ bottom view) of the three-layer molecular tribological system at (a) $\alpha=0.3$, (b) $\alpha=0.5$, and (c) $\alpha=0.8$. The fluid atoms in the second and the third layers are represented by the open red spheres and squares, respectively. The wall, the elastic substrate, and the fluid atoms in the first layer are represented by the green, blue, and filled red spheres, respectively. The coarse-grained far region of the substrate is represented by the gray spheres.

thickness decreases. The loading on the system accelerates the intermolecular ordering formation in the film.

The dependence of the maximum friction force $T_{x z}^{*} A$ (the product of the maximum shear stress $T_{x z}^{*}$ and the surface area $A$ ) on a normal force $-T_{z z} A$ represents the systematic tribological properties. Figure 14 displays the maximum shear stress $T_{x z}^{*}$ vs. the load $-T_{z z}$ curves for the mono-layer, two-layer, and three-layer film systems. At a fixed load, $T_{x z}^{*}$ of the mono-layer film system is remarkably larger than the two-layer and three-layer film systems. $T_{x z}^{*}$ of the two-layer film system is slightly larger than the three-layer film system. For the three cases, $T_{x z}^{*}$ rises almost linearly as the load $-T_{z z}$ increases. According to the AmontonsCoulomb friction law, the systematic static friction coefficient is defined as $\mu=-T_{x z}^{*} A / T_{z z} A=-T_{x z}^{*} / T_{z z}$. For the mono-layer film system, even the load $-T_{z z}$ approaches zero, and a large intermolecular interaction between the two solid surfaces would be overcome as they relatively slide, i.e., $T_{x z}^{*}>0$. Therefore, the mono-layer molecular thin-film lubrication does not satisfy the Amontons-Coulomb friction law as demonstrated in Fig. 14. However, for the two-layer and the three-layer film systems, since the thicker film prevents the intermolecular interaction between 


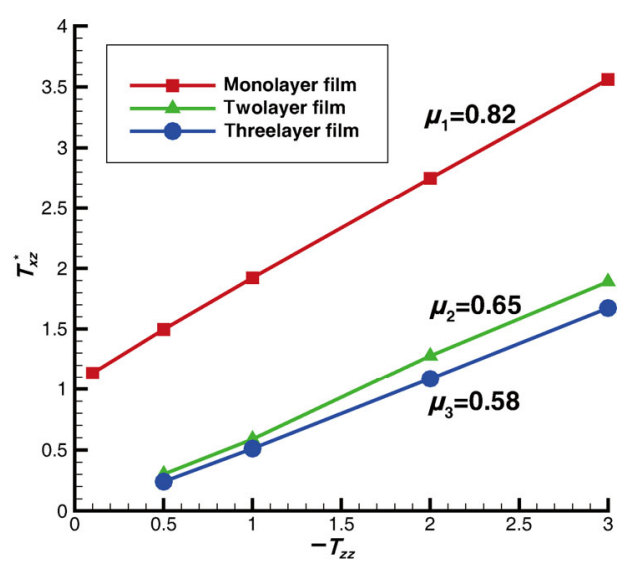

Fig. 14 The maximal shear stress $T_{x z}^{*}$ vs. the load $-T_{z z}$ for the mono-layer, two-layer, and three-layer fluid films. The systematic static friction coefficient $\mu_{l}$ of the $l$-layer fluid film is given.

two solid surfaces, they can approximately satisfy the Amontons-Coulomb friction law as shown in Fig. 14. To generate a uniform law to represent the dependence of $T_{x z}^{*}$ on $-T_{z z}$ for the three cases, an improved systematic static friction coefficient is proposed as $\mu=-\Delta T_{x z}^{*} A / \Delta T_{z z} A=-\Delta T_{x z}^{*} / \Delta T_{z z}$. Using this revised definition, we determined that $\mu_{1}=0.82, \mu_{2}=0.65$, and $\mu_{3}=0.58$ for the mono-layer, two-layer, and threelayer film systems, respectively. The systematic static friction coefficient retains a monotonously decreasing trend. It illustrates the function of reducing the friction and the wear of the multi-layer molecular thin-film lubrication.

\section{Conclusions and discussion}

This study investigated the effects of the elastic substrate on the tribological properties of 3D monolayer molecular thin-film lubrication. This study also proposes a multi-scale method, which combines an atomistic description of the near region with a coarse-grained description of the far region of the solid substrate to treat the thin-film lubrication. The coarse-graining reduces the original Hamiltonian of the far region to an effective one, which not only governs the nodes of the finite element mesh as pseudo-atoms, but also takes into account the harmonic contributions of the non-nodal atoms in the finite element mesh. The multi-scale method is applied to simulate the reversible shearing of the thin-film lubrication and it is compared to its several appro- ximation versions that are related to the substrate. The validity of the Amontons-Coulomb friction law on the multi-layer molecular thin-film lubrication was further tested with the multi-scale simulation based on the hybrid scheme. The conclusions of the investigations are summarized as follows:

1) By completely neglecting the elastic substrate (the wholly rigid treatment of the substrate or the crystalline model), this misses the snapping character in the transition process of the thin-film lubrication. This occurs with the elastic substrate (fully atomistic version of the system) and the hysteresis loop for the shearstress profile in the forward and backward processes.

2) This study introduced the concept of partial elasticity near the fluid-solid interface, which can improve the shape of the shear-stress profile of the thin-film lubrication. Only when the molecular layers of the elastic substrate are thick enough, the tribological properties of the system are qualitatively consistent with those for a fully atomistic version of the thin-film lubrication.

3) The shear-stress and the mean-separation profiles are determined by using the multi-scale method, which are in excellent agreement with those obtained from the fully atomistic simulation. The elastic response of the substrate can be effectively rendered in the hybrid scheme. The multi-scale method can successfully predict the tribological properties of the thin-film lubrication system for a range of parameters (temperature and film-substrate coupling strength), and significantly reduce the computing time and increase the computational efficiency.

4) The systematic static friction coefficient monotonously decreases as the molecular layer thickness in the fluid film increases. In comparison with the monolayer molecular thin-film lubrication, the multi-layer molecular thin-film lubrication plays a role in reducing the friction and wear of the system.

We emphasize that all of the above results pertain to the simplified 3D thin-film lubrication system that involves only two-body interactions and is subject to homogeneous isotropic deformations. The multi-scale method can be directly applied to some realistic models such as the sliding of two metal substrates (e.g., copper and iron) that are lubricated by lubricants (e.g., water and heptane). For more realistic models of the materials 
(e.g., mica and silicon), multiscale treatments of their sliding processes need to be determined.

\section{Acknowledgements}

This research is supported by the National Natural Science Foundation of China (Grants Nos. 11172310 and 11472284) and the Chinese Academy of Sciences (CAS) Strategic Priority Research Program (XDB22040403). The author thanks the National Supercomputing Center in Tianjin, China for assisting in the computation.

Open Access This article is licensed under a Creative Commons Attribution 4.0 International Li-cense, which permits use, sharing, adaptation, distribution and reproduction in any medium or for-mat, as long as you give appropriate credit to the original author(s) and the source, provide a link to the Creative Commons licence, and indicate if changes were made.

The images or other third party material in this article are included in the article's Creative Commons licence, unless indicated otherwise in a credit line to the material. If material is not in-cluded in the article's Creative Commons licence and your intended use is not permitted by statutory regulation or exceeds the permitted use, you will need to obtain permission directly from the copyright holder.

To view a copy of this licence, visit http://creativecommons.org/licenses/by/4.0/.

\section{References}

[1] Persson B N J. Sliding Friction: Physical Principles and Applications. Berlin (Germany): Springer, 1998.

[2] Luo J B, Hu Y Z, Wen S Z. Physics and Chemistry of Micro-/ Nanotribology. Maryland (USA): ASTM International, 2008.

[3] Zhao Y P. Nano and Mesoscopic Mechanics. Beijing (China): Science Press, 2014.

[4] Gnecco E, Meyer E. Elements of Friction Theory and Nanotribology. Cambridge (UK): Cambridge University Press, 2015.

[5] Schoen M, Rhykerd C L, Diestler D J Jr, Cushman J H. Shear forces in molecularly thin films. Science 245(4923): 1223-1225 (1989)

[6] Thompson P A, Robbins M O. Origin of stick-slip motion in boundary lubrication. Science 250(4982): 792-794 (1990)
[7] He G, Müser M H, Robbins M O. Adsorbed layers and the origin of static friction. Science 284(5420): 1650-1652 (1999)

[8] Gao G T, Zeng X C, Diestler D J. Nonlinear effects of physisorption on static friction. $J$ Chem Phys 113(24): 11293-11296 (2000)

[9] Hu Y Z, Wang H, Guo Y, Zheng L Q. Simulation of lubricant rheology in thin film lubrication Part I: Simulation of Poiseuille flow. Wear 196(1-2): 243-248 (1996)

[10] Hu Y Z, Wang H, Guo Y, Shen Z J, Zheng L Q. Simulation of lubricant rheology in thin film lubrication Part 2: Simulation of Couette flow. Wear 196(1-2): 249-453 (1996)

[11] Adamson A W. Physical Chemistry of Surfaces. 3rd ed. New York (USA): Wiley, 1976.

[12] Landman U, Luedtke W D, Ribarsky M W. Structural and dynamical consequences of interactions in interfacial systems. J Vac Sci Technol A 7(4): 2829-2839 (1989)

[13] Luan B, Robbins M O. The breakdown of continuum models for mechanical contacts. Nature 435(7044): 929-932 (2005)

[14] Müser M H. Elasticity does not necessarily break down in nanoscale contacts. Tribol Lett 67(2): 57 (2019)

[15] Vakis A I, Yastrebov V A, Scheibert J, Nicola L, Dini D, Minfray C, Almqvist A, Paggi M, Lee S, Limbert G, et al. Modeling and simulation in tribology across scales: An overview. Tribol Int 125: 169-199 (2018)

[16] Kohlhoff S, Gumbsch P, Fischmeister H F. Crack propagation in b.c.c. crystals studied with a combined finite-element and atomistic model. Philos Mag A 64(4): 851-878 (1991)

[17] O'Connell S T, Thompson P A. Molecular dynamicscontinuum hybrid computations: A tool for studying complex fluid flows. Phys Rev E 52(6): R5792-R5795 (1995)

[18] Singharoy A, Joshi H, Ortoleva P J. Multiscale macromolecular simulation: Role of evolving ensembles. J Chem Inf Model 52(10): 2638-2649 (2012)

[19] Phillips R, Dittrich M, Schulten K. Quasicontinuum representations of atomic-scale mechanics: From proteins to dislocations. Annu Rev Mater Res 32: 219-233 (2002)

[20] Tadmor E B, Ortiz M, Phillips R. Quasicontinuum analysis of defects in solids. Philos Mag A 73(6): 1529-1563 (1996)

[21] Shenoy V B, Shenoy V, Phillips R. Finite temperature quasicontinuum methods. Mater Res Soc Symp Proc 538: 465-471 (1999)

[22] Diestler D J, Wu Z B, Zeng X C. An extension of the quasicontinuum treatment of multiscale solid systems to nonzero temperature. J Chem Phys 121(19): 9279-9282 (2004)

[23] Wu Z B, Diestler D J, Zeng X C. Multiscale treatment of thin-film lubrication. Mol Simul 31(12): 811-815 (2005) 
[24] Wu Z, Zeng B X C. Multiscale simulation of thin-film lubrication: Free-energy-corrected coarse graining. Phys Rev E 90(3): 033303 (2014)

[25] Luan B Q, Hyun S, Molinari J F, Bernstein N, Robbins M O. Multiscale modeling of two-dimensional contacts. Phys Rev E 74(4): 046710 (2006)

[26] Luan B Q, Robbins M O. Hybrid atomistic/continuum study of contact and friction between rough solids. Tribol Lett 36(1): 1-16 (2009)

[27] Ramisetti S B, Anciaux G, Molinari J F. MD/FE multiscale modeling of contact. In Fundamentals of Friction and Wear on the Nanoscale. Gnecco E, Meyer E, Eds. Cham: Springer, 2015: 289-312.

[28] LeSar R, Najafabadi R, Srolovitz D J. Finite-temperature defect properties from free-energy minimization. Phys Rev

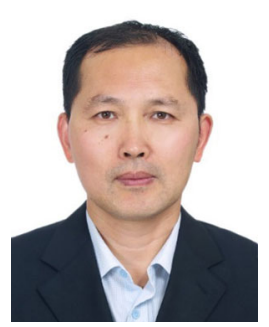

Zuobing WU. He received his Ph.D. degree in theoretical physics from Chinese Academy of Sciences, Beijing, China in 1995. He worked as a postdoc in Physical College at Peking University during 1996-1997 and a visiting scholar in Chemical
Lett 63(6-7): 624-627 (1989)

[29] Schoen M, Diestler D J, Cushman J H. Shear melting of confined solid monolayer films. Phys Rev B 47(10): 5603-5613 (1993)

[30] Schoen M, Hess S, Diestler D J. Rheological properties of confined thin films. Phys Rev E 52(3): 2587-2602 (1995)

[31] Schrodinger E. Statistical Thermodynamics. New York (USA): Dover, 1989.

[32] Hill T. An Introduction to Statistical Thermodynamics. New York (USA): Dover, 1986.

[33] Allen M P, Tildesley D J. Computer Simulation of Liquids. Oxford (UK): Clarendon, 1987.

[34] Diestler D J, Gao G T, Zeng X C. Role of hysteresis in the molecular picture of friction. Phys Chem Chem Phys 3(7): 1175-1178 (2001)

Department at University of Nebraska-Lincoln during 2002-2004, respectively. As a permanent faculty, he joined the State Key Laboratory of Nonlinear Mechanics at Institute of Mechanics, Chinese Academy of Sciences from 1998. His current position is a research professor. His research areas cover multi-scale mechanics, interfacial fluid mechanics, and nonlinear mechanics. 